\title{
BREAST CANCER AND ITS IMPACT IN SOCIETY AT A GLANCE
}

\author{
George Dimitrov*,1, Feodor Odzhakov* and George Baytchev ${ }^{\dagger}$ \\ ${ }^{*}$ Medical University of Sofia, Bulgaria, ${ }^{\dagger}$ Military Medical Academy of Sofia, Bulgaria
}

\begin{abstract}
ABTRACT
The first in a series of reviews discusses the literature published so far relating to breast cancer including: epidemiology of the disease, economic impact, pathology of breast cancer as it remains the most common cancer diagnosed in women, the main molecular mechanisms of tumorigenesis accepted today, the invasion \& metastasis cascade and the concerning relationship between benign and malignant disease.
\end{abstract}

KEYWORDS breast cancer; breast disease; breast cancer impact

\section{Epidemiology}

Breast cancer is the most common occurring malignancy in women of the developed world, comprising almost a third of all malignancies in females. It is defined as a malignant proliferation of the epithelial cells from which the breast ducts and milk-producing lobules constitute. The latest Western European statistics have shown that on average about 100,000 incidents of invasive breast carcinoma and, though slowly declining, 25,000 deaths due to the subsequent late stage metastatic spread occur annually (European region) [1]. On the other hand the incidents of the disease in eastern European countries were found to be somewhat lower, though still demonstrating a significant mortality factor [2], and unfortunately as the research shows there has been no significant improvement in breast cancer morbidity rates in that region.

Globally, the lifetime risk of a woman developing invasive breast cancer is $13 \%$, Meaning that one out of eight females will develop breast cancer at some point in their life [4]. The mortality rates have been shown to be highest in the ancient $(>75$

Copyright (c) 2015 by the Bulgarian Association of Young Surgeons

DOI: $10.5455 /$ ijsm. 188450

First Received: May 11, 2015

Published Online: May 20, 2015

Manuscript Associate Editor: Efstratios Koutroumpakis (USA)

Editor-in Chief: Ivan Inkov (BG)

Reviewer: Iskra Daskalova (BG)

${ }^{1}$ George Dimitrov, Medical University of Sofia, Blvd. „Academic Ivan Geshov“ 15, 1431

Sofia, Bulgaria

Email: george121@gmail.com years of age) and sadly, also in the very young ( $<35$ years of age) [5]. This may be explained by the fact that the more aggressive disease has been observed to manifest in the very young in contrast to the very old, to whom the prescribed treatments may be substandard and not aggressive enough, or may present with co-morbid disease that increases fatality [6].

Even though, statistically, recurrence has been shown in the first three years after treatment, the chance of recurrent cancer can exist up to twenty years [7]. These rates are especially observed in the HER2 positive or triple negative (ER -ive, $\mathrm{PgR}$-ive and HER 2 -ive) lesions (about $60 \%-80 \%$ of the cases), but not so evident in the ER-positive cohort, which generally tend to be late.

\section{Economic Impact of Breast Cancer}

The high morbidity and mortality this disease is associated with (Fig. 1) and the subsequent patient care, has had and will continue having, a toll on the world's economy in more than one way.

Firstly, cancer care cost the EU about $€ 126$ billion in 2009 , out of which breast cancer care represented a significant segment, $€ 13.2$ billion, of all the cancer types treated in that year [8].

Secondly, the person's lost years of life and productivity caused by cancer represents the single largest drain on the global economy.

This economic impact of the disease is a severe problem that can be tackled by quick action through early diagnosis and treatment, only then we will be able to avert needless deaths and suffering caused by cancer and to reduce its devastating economic impact. However, to accomplish this, we have to fight the emperor of all ailments which is not an easy feat. 
1.

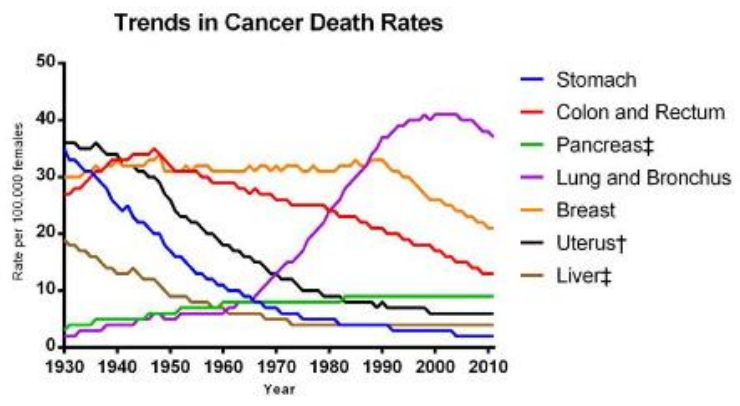

Fig. 1: Trends in cancer death rates worldwide by the age of $65,1930-2011$. Rates are per 100,000 age-adjusted to the 2000.As seen here breast cancer is 2 nd only to lung cancer death rates in females. Created using Graphpad PrismTM and the original data from US Mortality Volumes 1930 to 1959, US Mortality Data 1960 to 2011, National Centre for Health Statistics, Centres for Disease Control and Prevention [3]. + refers to the combination of uterine cervix and uterine corpus, $\ddagger$ evident increase in mortality rates.

Fortunately with the advancement of molecular and cell biology we have been able to achieve much progress in the war against cancer, by elucidating some of the pathways cancer cells deregulate in order to further their advancement $[9,10]$.

\section{Pathogenesis}

Most breast cancers ( $90 \%$ ) are carcinomas meaning, thy rise from epithelial elements of the breast parenchyma. In the case of breast cancer, carcinomas are divided into two major groups: in situ carcinomas which can arise from either duct (Fig. 2) or lobular epithelium yet confined there with not protruding through the basement membrane, and infiltrating carcinomas of either origin which invade through this barrier membrane to underlying tissue (Fig 3.).

Most common tumours are of ductal origin followed by tumours of lobular origin which often present as multi-focal aggregates and not necessarily as a single solid lump. Metastasis which was previously mentioned as the main morbidity factor, is generally a result of infiltrating carcinomas which gives the potential to tumour cells to migrate to other sites and unfortunately and at the ultimate price, the life of the patient [11].

Regarding the actual tumorigenesis, one of the main pathways frequently found aberrantly activated in a significant amount of cancers is the mitogen-activated protein kinase (MAP$\mathrm{K})$ cascade, and breast cancer is in this category [12].

The MAP-K family consists of seven classes through which cells can obtain uncontrollable division and growth (Fig 4.) [13]. In breast cancer, only four of these cascades have been found responsible for tumorigenesis; the extracellular regulated kinase ERK1/2 pathway, the ERK5 pathway, the p38 pathway and the c-Jun N-terminal kinase (JNK) pathway. Signalling through the ERK1/2 pathway, in particular, has been implicated as being crucial [14, 15].
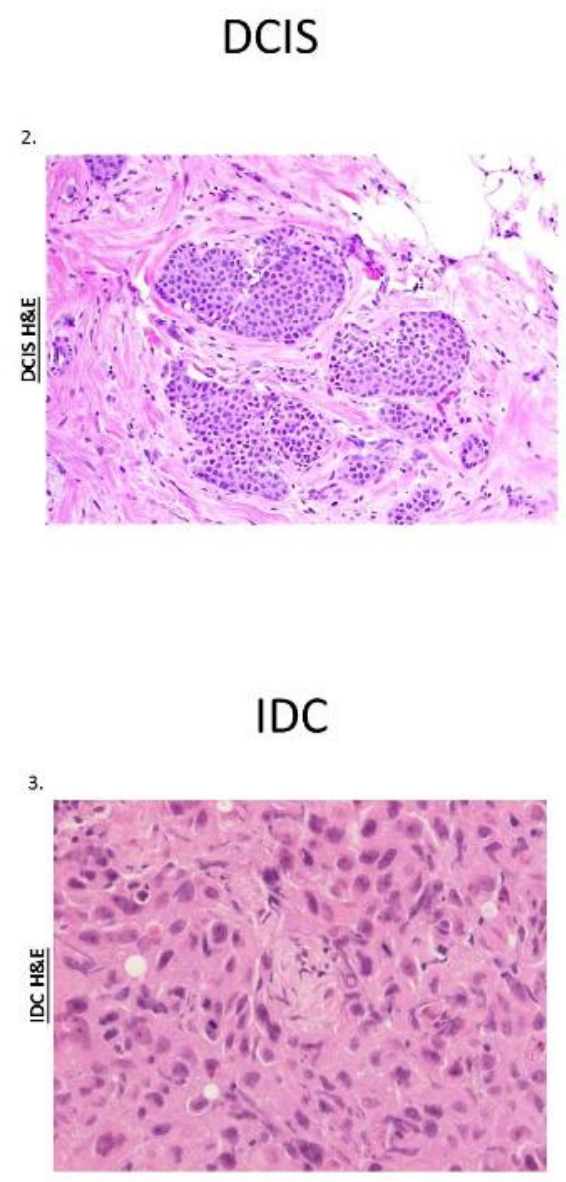

Fig. 2; Fig. 3 Represent a micrograph of both a ductal carcinoma in situ (DCIS) and invasive ductal carcinoma (IDC) respectively. We can clearly see that in DCIS the tumour cells have not infiltrated through the basement membrane.

Analysis performed at the department of Histopathology, Imperial College, Charing-Cross Hospital, London, under supervision of Prof. Smai Shousha. Magnification; X200-300, respectively. 


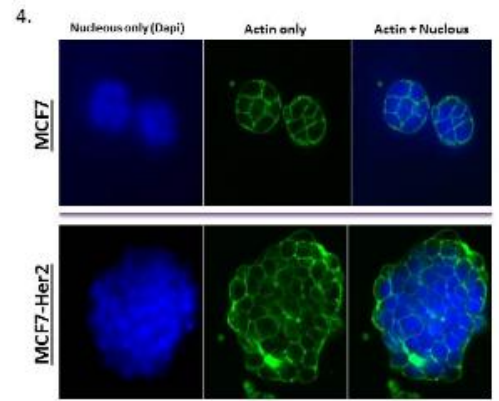

Fig. 4: MCF7 cells line grown as a 3D culture on matri-gel and immunoflourescently stained using an antibody against F-actin. Cells stably transfected with Her2/neu have demonstrated forming much larger and more deformed (acinar looking) colonies than similarly grown non transfected cells. Dapi dye was utilized for nuclear staining and GFP for Actin; visualized under a fluorescence microscope. Her2/Neu has been found to interact with the MAPK pathway hence we can explain how the more aggressive phenotype emerges. Analysis performed at the department of Oncology and Surgery, Imperial College, Hammersmith Campus, London.

Also, molecules such as Her2/neu, which is found overexpressed in 30\% of breast cancers [16], have been found to deregulate such pathways. Moreover, Her2/neu has been shown to oppose apoptosis through regulation of the PI3K pathway [17]. The acquisition of a more invasive phenotype can also be achieved through the deregulation of these signalling pathways achieved by modulation of various proteolytic enzymes, such as the matrix metalloproteinases (MMPs), which degrade the basal and reticular laminas thus promote cell migration. The latter has been shown to be encouraged by high ERK1/2 activity [18, 19].

In addition genetic alterations in the BRCA1/BRCA2 genes, which are responsible for coding repair proteins for doublestrand DNA breaks, and their associated chaperon BARD1 have been strongly linked with breast cancer development, aggressiveness of the phenotype and patient prognosis [20].

The interesting aspect of the relationship between benign and malignant disease is of great concern not only for doctors and patients but also insurance companies, as there are different prerequisites some of which may confer a definitely elevated risk of malignization while others may confer no risk at all. Breast lesions that possess no proliferative changes represent the cohort of specimens conferring no significant malignization risk [21]. Examples of such lesions include benign solid tumours that may contain glandular as well as loose connective tissue, simple fibroadenomas and ductal ectasia. Though some papillomas, especially micro-papillary tumours, can be considered to possess an elevated malignization risk, it has been shown that solitary papillomas (non-micro-papillary type) belong to the lesions conferring no elevated risk [22].

Fibrocystic disease which can be sub-divided into (stromal) hyperplasia, adenosis and cystic changes as a whole has been

\section{Metastasis schematic}

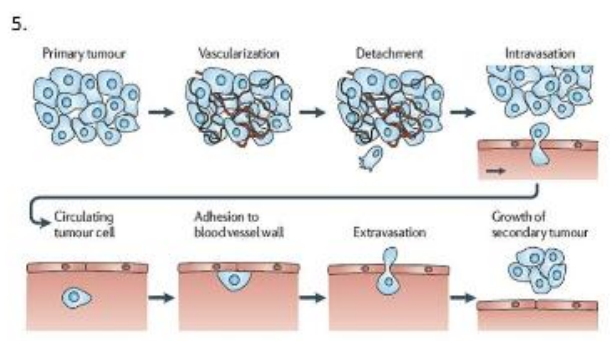

Fig. 5: A schematic diagram of the metastasis process which involves cells detaching from the primary tumour, entering the blood or lymph circulation and eventually exiting and reseeding in the secondary site. Schematic courtesy of Writz et al. [15].

shown to not carry an elevated risk to develop cancer (other than the potential for missing an already present malignant mass) [23].

The most common encountered lesion of the breast that possesses a definite elevated malignization risk is the ductal hyperplasia without atypia, which confers about a 2 -fold increase in developing invasive cancer. It is believed that the loss of TGFbeta receptor 2 in the mammary epithelium handles the elevated risk [24, 25]. Atypical hyperplasia, on the other hand, characterized by the cells losing their apical-basal orientation, confers a 4-fold elevated risk for malignization. Unless there is one or more family relative diagnosed with this disease that raises the risk to 6-fold. Other benign lesions that confer a 2-fold elevated risk for malignization include sclerosing adenosis, diffused papillomatosis and fibroadenomas with a proliferative disease that may present as tumours containing $3 \mathrm{~mm}$ in diameter or greater cysts $[26,27]$.

Nipple discharge and breast pain are a sign of great concern among patients relating to invasive cancer. Though, it has been shown that non-bloody discharge and even bilateral discharge are often signs of benign disease. Even if present in invasive disease they are usually unilateral and associated with a palpable mass [28]. Similarly, breast pain is an uncommon presentation in breast cancer $[28,29]$.

\section{Metastasis}

As mentioned metastatic spread, mainly to vital organs, is the main culprit for cancer mortality rates. This complex stochastic event involves cells deserting the primary tumour mass and circulating to a different site via the blood and lymphatic systems. Thus resulting in the formation of new colonies once these migrating cells reach the novel tissue parenchyma (Fig. 5) [30, 31]. It has been shown that breast cancer often gives rise to metastatic colonies promiscuously in a variety of tissues, including: lungs, liver, bones and the brain [32], and these nomadic cancer cells are the most dangerous manifestations of the oncogenic process. Therefore, early detection and treatment of breast cancer is of great importance. 
MCF-7 stained for F-actin

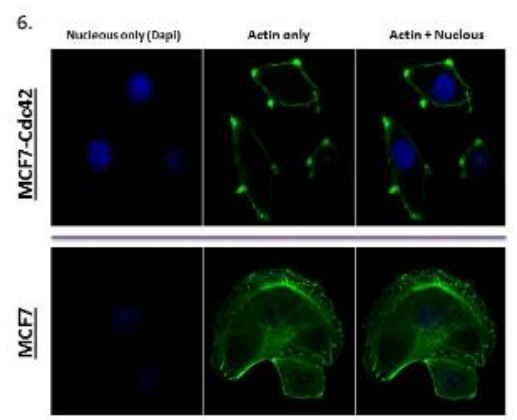

Fig. 6: Cdc42 transient overexpression in MCF7 cell line induces changes in the actin cytoskeleton which in turn enhances the aggressiveness of cells - The figure shows F-actin localizing to the cell edge (lammeliapodium) as actin rich protrusions in the transfected and overexpressed cell line - As seen from the analysis on monolayer grown samples. Dapi dye was utilized for nuclear staining and GFP for Actin; visualized under a fluorescence microscope. Analysis performed at the department of Oncology and Surgery, Imperial College, Hammersmith Campus, London.

It is accepted that metastatic spread relies on the aberrant activation of various signalling cascades.

Kinases have been assigned a significant role in the involved signal transductions of these cascades, and it has been shown that this holds true especially in pathways associated with cell proliferation and motility [33]. Some of the primary pathways implicated in the promotion of tumour and metastasis progression solely or through cross-talk with other effectors such as Ras involve the family of Rho-GTPases [34].

This family is a part of the Ras superfamily of GTPases, and in similar manner changes between an active and inactive state occur when bound to GTP or GDP respectively.

The Rho family have been linked to the regulation of transcription, proliferation, and migration through reorganization of the actin cytoskeleton - integrins, which are crucial to cytoskeleton modification have also been implicated in advancing cancer invasion [35-37].

The best-characterized molecules include Rho proper that regulates stress fibres and focal adhesion formation, and Race \& Cdc42, which regulate lamellipodium and filopodium formation, respectively [38]. Due to the various key functions Rho proteins regulate, it is of no surprise that they have significant roles in tumour biology. In addition, Rho proteins overexpression and/or hyperactivation, which can be observed in a large variety of human tumours, further solidified these molecules' involvement in the cancer initiation and progression cascade (Fig 6.).

\section{Conclusion}

Although breast cancer is an important cause of morbidity and mortality, the rapid advancements in diagnostic and therapeutic modalities, in addition to patient education, brought a continuous evident reduction in the patient's morbidity and mortality rates.
To this day, the most effective treatment is a combination of local and systemic therapies. However, to further increase the patient's well-being, primary (neoadjuvant) chemotherapy was introduced. This type of pre-surgical modality has been implemented in order to achieve down-staging of the tumour mass, thus reducing the extent of mastectomy in favour of breastconserving therapies such as wide local excisions [39]. Moreover, it has been shown that not only patient with advanced disease respond well to this type of treatment, but also patients who were diagnosed with small, early stage, tumours $(<2 \mathrm{~cm})$ [40]. However, only a strong comprehension of all the issues described briefly here and in other literature, will help elucidate what now is called incurable disease, which may arise from further genetic and/or epigenetic changes.

This concludes this issues topic, next we will explore the aforementioned therapeutic modalities of breast cancer and their subsequent consequences on the patient's quality of life.

\section{Authors' Statements}

Competing Interests

The authors declare no conflict of interest.

\section{References}

1. Ferlay, J., et al., Cancer incidence and mortality patterns in Europe: estimates for 40 countries in 2012. Eur J Cancer, 2013. 49(6): p. 1374-403.

2. Znaor, A., et al., Cancer incidence and mortality patterns in South Eastern Europe in the last decade: gaps persist compared with the rest of Europe. Eur J Cancer, 2013. 49(7): p. 1683-91.

3. Jemal, A., et al., Cancer statistics, 2010. CA Cancer J Clin, 2010. 60(5): p. 277-300.

4. DeSantis, C., et al., Breast cancer statistics, 2013. CA Cancer J Clin, 2014. 64(1): p. 52-62.

5. Smith, H.O., et al., Hormone replacement therapy in the menopause: a pro opinion. CA Cancer J Clin, 1996. 46(6): p. 343-63.

6. Warnberg, F., et al., Risk factors for subsequent invasive breast cancer and breast cancer death after ductal carcinoma in situ: a population-based case-control study in Sweden. Cancer Epidemiol Biomarkers Prev, 2001. 10(5): p. 495-9.

7. Cossetti, R.J., et al., Comparison of breast cancer recurrence and outcome patterns between patients treated from 1986 to 1992 and from 2004 to 2008. J Clin Oncol, 2015. 33(1): p. 65-73.

8. Luengo-Fernandez, R., et al., Economic burden of cancer across the European Union: a population-based cost analysis. Lancet Oncol, 2013. 14(12): p. 1165-74.

9. Sikora, K., Molecular biology and the treatment of cancer. Clin Radiol, 1985. 36(2): p. 117-24.

10. Busch, H., A general concept for molecular biology of cancer. Cancer Res, 1976. 36(11 Pt. 2): p. 4291-4. 
11. Korhonen, T., et al., The impact of lobular and ductal breast cancer histology on the metastatic behavior and long term survival of breast cancer patients. Breast, 2013. 22(6): p. 1119-24.

12. Dhillon, A.S., et al., MAP kinase signalling pathways in cancer. Oncogene, 2007. 26(22): p. 3279-90.

13. Krishna, M. and H. Narang, The complexity of mitogenactivated protein kinases (MAPKs) made simple. Cell Mol Life Sci, 2008. 65(22): p. 3525-44.

14. Whyte, J., et al., Key signalling nodes in mammary gland development and cancer. Mitogen-activated protein kinase signalling in experimental models of breast cancer progression and in mammary gland development. Breast Cancer Res, 2009. 11(5): p. 209.

15. Haagenson, K.K. and G.S. Wu, The role of MAP kinases and MAP kinase phosphatase- 1 in resistance to breast cancer treatment. Cancer Metastasis Rev, 2010. 29(1): p. 143-9.

16. Mitri, Z., T. Constantine, and R. O'Regan, The HER2 Receptor in Breast Cancer: Pathophysiology, Clinical Use, and New Advances in Therapy. Chemother Res Pract, 2012. 2012: p. 743193.

17. Munster, P.N., et al., Degradation of HER2 by ansamycins induces growth arrest and apoptosis in cells with HER2 overexpression via a HER3, phosphatidylinositol 3'-kinaseAKT-dependent pathway. Cancer Res, 2002. 62(11): p. 31327.

18. Reddy, K.B., S.M. Nabha, and N. Atanaskova, Role of MAP kinase in tumor progression and invasion. Cancer Metastasis Rev, 2003. 22(4): p. 395-403.

19. Krueger, J.S., et al., Temporal and quantitative regulation of mitogen-activated protein kinase (MAPK) modulates cell motility and invasion. Oncogene, 2001. 20(31): p. 4209-18.

20. Wu, J.Y., et al., Aberrant expression of BARD1 in breast and ovarian cancers with poor prognosis. Int J Cancer, 2006. 118(5): p. 1215-26.

21. Dyrstad, S.W., et al., Breast cancer risk associated with benign breast disease: systematic review and meta-analysis. Breast Cancer Res Treat, 2015. 149(3): p. 569-75.

22. Moon, H.J., et al., Breast papilloma without atypia and risk of breast carcinoma. Breast J, 2014. 20(5): p. 525-33.

23. Dupont, W.D., et al., Long-term risk of breast cancer in women with fibroadenoma. N Engl J Med, 1994. 331(1): p. $10-5$.

24. Gobbi, H., et al., Transforming growth factor-beta and breast cancer risk in women with mammary epithelial hyperplasia. J Natl Cancer Inst, 1999. 91(24): p. 2096-101.

25. Novitskiy, S.V., et al., TGF-beta receptor II loss promotes mammary carcinoma progression by Th17 dependent mechanisms. Cancer Discov, 2011. 1(5): p. 430-41.

26. Jacobs, T.W., et al., Radial scars in benign breast-biopsy specimens and the risk of breast cancer. N Engl J Med, 1999. 340(6): p. 430-6.
27. Collins, L.C., et al., Magnitude and laterality of breast cancer risk according to histologic type of atypical hyperplasia: results from the Nurses' Health Study. Cancer, 2007. 109(2): p. 180-7.

28. Meshkat, B., et al., A comparison of clinical-pathological characteristics between symptomatic and interval breast cancer. Breast, 2015. 24(3): p. 278-82.

29. Duijm, L.E., et al., Value of breast imaging in women with painful breasts: observational follow up study. BMJ, 1998. 317(7171): p. 1492-5.

30. Klein, C.A., Cancer. The metastasis cascade. Science, 2008. 321(5897): p. 1785-7.

31. Wirtz, D., K. Konstantopoulos, and P.C. Searson, The physics of cancer: the role of physical interactions and mechanical forces in metastasis. Nat Rev Cancer, 2011. 11(7): p. $512-22$.

32. Lacroix, M., Significance, detection and markers of disseminated breast cancer cells. Endocr Relat Cancer, 2006. 13(4): p. 1033-67.

33. Kozlowski, J., A. Kozlowska, and J. Kocki, Breast cancer metastasis - insight into selected molecular mechanisms of the phenomenon. Postepy Hig Med Dosw (Online), 2015. 69: p. $447-51$.

34. Vega, F.M. and A.J. Ridley, Rho GTPases in cancer cell biology. FEBS Lett, 2008. 582(14): p. 2093-101.

35. Parri, M. and P. Chiarugi, Rac and Rho GTPases in cancer cell motility control. Cell Commun Signal, 2010. 8: p. 23.

36. Xu, Y., et al., The kinase LMTK3 promotes invasion in breast cancer through GRB2-mediated induction of integrin beta(1). Sci Signal, 2014. 7(330): p. ra58.

37. Inuzuka, M., et al., [Integrins involved in tumor invasion and metastasis]. Nihon Rinsho, 1995. 53(7): p. 1631-6.

38. Etienne-Manneville, S. and A. Hall, Rho GTPases in cell biology. Nature, 2002. 420(6916): p. 629-35.

39. Bonadonna, G., et al., Primary chemotherapy in operable breast cancer: eight-year experience at the Milan Cancer Institute. J Clin Oncol, 1998. 16(1): p. 93-100.

40. Van der Hage, J.A., et al., Preoperative chemotherapy in primary operable breast cancer: results from the European Organization for Research and Treatment of Cancer trial 10902. J Clin Oncol, 2001. 19(22): p. 4224-37. 